\title{
Evaluation of the efficacy of computed tomographic coronary angiography in assessing coronary artery morphology and physiology: rationale and study design
}

\author{
Anantharaman Ramasamy,1,2 MBChB MRCP; Hannah Safi,1,3 PhD; James C Moon,1,4 MD; Mervyn \\ Andiapen,1 RN; Krishnaraj S Rathod,1,2 MD PhD; Pal Maurovich-Horvat,5 MD PhD; Retesh Bajaj,1,2 \\ MBChB MRCP; Patrick W Serruys,6 MD PhD; Anthony Mathur,1,2 MD; Andreas Baumbach,1,2 MD; \\ Francesca Pugliese,1,2 MD PhD; Ryo Torii, 3 PhD; Christos V Bourantas,1,2,4,* MD PhD
}

\author{
1 Department of Cardiology, Barts Heart Centre, Barts Health NHS Trust, London, UK \\ 2 William Harvey Research Institute, Queen Mary University London, UK \\ 3 Department of Mechanical Engineering, University College London, London, UK \\ 4 Institute of Cardiovascular Sciences, University College London, London, UK \\ 5 MTA-SE Lendulet Cardiovascular Imaging Research Group, Heart and Vascular Centre, Semmelweis \\ University, Budapest, Hungary \\ 6 Faculty of Medicine, National Heart \& Lung Institute, Imperial College London, UK
}

Short title: computed tomographic coronary angiography plaque assessment

\section{*Address for correspondence}

Christos Bourantas, $\mathrm{MD}, \mathrm{PhD}$

Consultant Cardiologist, Barts Heart Centre,

Barts Heart Centre, West Smithfield, London EC1A 7BE

E-mail: cbourantas@gmail.com

Phone: +44 2073777000

Fax: +44 2077919670

Keywords: Computed tomographic coronary angiography, near infrared spectroscopy-intravascular imaging, atherosclerosis, local haemodynamic forces 


\begin{abstract}
Computed tomographic coronary angiography (CTCA) is a non-invasive imaging modality which allows plaque burden and composition assessment and detection of plaque characteristics associated with increased vulnerability. In addition, CTCA-based coronary artery reconstruction enables local haemodynamic forces assessment, which regulate plaque formation and vascular inflammation and prediction of lesions that are prone to progress and cause events. However, the use of CTCA for vulnerable plaque detection in the clinical arena remains limited. To unlock the full potential of CTCA and enable its broad use, further work is needed to develop user-friendly processing tools that will allow fast and accurate analysis of CTCA, computational fluid dynamic modelling and evaluation of the local hemodynamic forces. The present study aims to develop a seamless platform that will overcome the limitations of CTCA and enable fast and accurate evaluation of plaque morphology and physiology. We will analyse imaging data from 70 patients with coronary artery disease who will undergo state-ofthe-art CTCA and near infrared spectroscopy-intravascular ultrasound imaging and develop and train algorithms that will take advantage of the intravascular imaging data to optimise vessel segmentation and plaque characterization. Furthermore, we will design an advanced module that will enable reconstruction of coronary artery anatomy from CTCA, blood flow simulation, shear stress estimation and comprehensive visualization of vessel pathophysiology. These advances are expected to facilitate the broad use of CTCA, not only for risk stratification but also for the evaluation of the effect of emerging therapies on plaque evolution.
\end{abstract}




\section{Introduction}

There is growing evidence that vessel physiology and plaque characteristics are able to detect high-risk plaques that are likely to progress and cause cardiovascular events.(1-3) Accurate in vivo assessment of plaque characteristics is traditionally performed by intravascular imaging. Today, three intravascular imaging modalities are available in the clinical arena [intravascular ultrasound (IVUS), optical coherence tomography (OCT), and near infrared spectroscopy (NIRS)-IVUS]. These have been studied in large prospective studies and showed that they can provide useful prognostic information and detect vulnerable lesions and high-risk patients that are likely to suffer a cardiovascular event. $(1,2,4)$ Histology studies have shown that NIRS-IVUS has an excellent accuracy in assessing plaque phenotype and it is the best modality for characterising its composition as it combines an ultrasound probe that gives information about plaque and calcific tissue burden and a NIRS probe that can accurately detect the lipid component. $(5,6)$ In addition the recently published Lipid Rich Plaque study has provide robust evidence that this modality enables detection of vulnerable plaques and patients and today NIRS-IVUS is the only FDA-approved imaging technique for the detection of plaques and patients that are at risk to suffer a cardiovascular event (2). Despite the potential of this modality in detecting vulnerable plaques, its use in the clinical arena for this purposes is limited. This is an invasive technique which can be only be used in patients undergoing coronary angiography. Intravascular imaging is associated with a risk of complications and it does not allow complete assessment of the entire coronary tree.

Non-invasive imaging and in particular, computed tomographic coronary angiography (CTCA) have appeared as a promising alternative for the study of atherosclerosis and seems to be able to overcome limitations of intravascular imaging. Cumulative evidence has shown that CTCA can detect lumen and vessel wall dimensions and quantify plaque burden and composition.(7-9) Moreover, recent reports have shown that computational fluid dynamic (CFD) analysis is feasible in CTCA-derived reconstructions and that the estimated local hemodynamic forces have a value in detecting segments that are likely to exhibit disease progression. $(10,11)$ In addition, large scale retrospective studies have shown that CTCA can identify vulnerable plaques and patients at risk of suffering cardiovascular events. Recently, the EMERALD study has demonstrated that plaque characteristics and the local 
hemodynamic forces estimated by CTCA have incremental value in differentiating lesions that caused a myocardial infarction from those that remained quiescent.(12) Despite these promising findings, CTCA is not used in everyday clinical practice for vulnerable plaque detection and patient risk stratification. This should be partially attributed to the increased time required for CTCA analysis as well as to the limitations of CTCA to accurately detect lumen and vessel wall borders and quantify plaque burden and composition - especially in calcified plaques. In the present study, we aim to develop and validate methodologies that will overcome the above limitations and enable the use of CTCA for accurate risk stratification and thus personalised secondary prevention.

\section{Study design}

\section{Study objectives}

The "Evaluation of the efficacy of computed tomographic coronary angiography in assessing coronary artery morphology and physiology" is a prospective observational multi-imaging single centre study. The primary objective of the study is to examine whether CTCA allows accurate assessment of coronary artery plaque phenotype using invasive imaging as the gold standard. Secondary objectives of the study are: 1) to develop segmentation algorithms and plaque characterisation methodologies that will enable accurate assessment of plaque burden and composition, 2) examine the efficacy of CTCA in assessing the local haemodynamic forces and 3) develop a seamless platform that will enable CTCA segmentation, plaque characterisation, blood flow simulation and evaluation of the endothelial shear stress (ESS) in less than $1 \frac{1}{2}$ hour per patient.

\section{Patient population}

Seventy patients with typical angina symptoms who had elective coronary angiography showing at least one obstructive lesion and are referred to Barts Heart Centre for further evaluation with a fractional flow reserve (FFR) study or percutaneous coronary intervention (PCI) will be considered for participation. The inclusion and exclusion criteria of the study are shown in Table 1. Patients who fulfil these criteria will be contacted by the research team and a copy of the patient information sheet will be provided. Patients who are keen to participate will be booked to attend two visits to the Barts Heart 
Centre for: 1) study consenting followed by CTCA and pre-assessment clinic visit and 2) a 3-vessel intravascular imaging study along with PCI as required. Following the procedure, patients will be transferred back to the day case ward for monitoring and they will be discharged later that day.

\section{Patient withdrawal}

Patients will be free to withdraw consent at their own request at any time without the need to provide reasons for their decision. Additionally, the principal investigator will be able to withdraw a patient from the study if there are concerns about patient safety and well-being. Withdrawals will be documented in the case report form and in the patients' medical records.

\section{Patient pre-assessment}

On the day of the pre-assessment visit, patient's fitness for invasive angiography will be assessed by trained nurses as per Barts Health NHS Trust protocols. Blood samples (20mls) will be obtained to check for full blood count, renal function and lipid profile. Patients cardiovascular risk factors, revascularisation history and current medications will be recorded.

\section{Computed tomography coronary angiography}

CTCA will be performed after consenting using a third generation dual-source scanner (Somatom Force, Siemens Healthineers, Forchheim, Germany). Before the CT scan all patients will receive sublingual nitroglycerin $(0.4 \mathrm{mg} / \mathrm{dose})$ and those with heart rate $>70$ beats per minute, will also receive intravenous metoprolol (maximum $40 \mathrm{mg}$ ), provided there are no contraindications. The CTCA scan parameters include prospective ECG-triggered sequential scan mode, gantry rotation time of $250 \mathrm{~ms}$, $128 \times 2 \times 0.5 \mathrm{~mm}$ collimation with z-flying focal spot for both detectors, minimum tube voltage of $100 \mathrm{kV}$ and tube current determined by the scanner. A bolus of iodinated contrast agent (Omnipaque 350) which varies between 65 or $78 \mathrm{mls}$ depending on the patient's body habitus will be injected intravenously at an injection rate of $4-5 \mathrm{ml} / \mathrm{s}$ followed by $32 \mathrm{mls}$ saline chaser with the same injection rate. A test bolus technique will be used to synchronise the start of image acquisition. All CT images will be digitally stored for offline analysis. 


\section{Intravascular imaging}

A senior interventional trainee and a consultant cardiologist will perform the coronary angiography according to Barts Health Trust protocols. The access route, choice of guide catheters and coronary wires will be left to the operators' discretion. Heparin (intracoronary or intravenous according to the operator's discretion) will be administered to maintain an ACT $>250$ throughout the procedure. All patients will receive 400 micrograms of intracoronary nitrate prior to image acquisition, provided there are no contraindications. Images will be acquired after optimal intubation of the guiding catheter; an effort will be made to avoid panning during angiography and acquire for each studied vessel at least two angiographic views, that are at least 25o apart, with good contrast opacification and minimal vessel foreshortening and overlapping. Before FFR or PCI - depending on the clinical need - NIRS-IVUS imaging will be performed in all the 3-epicardial vessels and their major side branches with diameter $\geq 2 \mathrm{~mm}$. NIRS-IVUS will be performed by the Makoto ${ }^{\mathrm{TM}}$ imaging system (Infraredx, Burlington, MA) The catheter will be advanced $5 \mathrm{~mm}$ distally to the most distal side branch that is visible in both angiography and CTCA imaging and will be pulled-back by a motorised pullback device that will withdraw the catheter at $0.5 \mathrm{~mm} / \mathrm{s}$; images will be acquired at 30 frames per second and digitally archived. Patients will then undergo pressure wire study or PCI as per clinical indication.

\section{Image segmentation}

Imaging data will be anonymised and analysed offline blinded to clinical details by an expert analyst using dedicated workstation. Anatomical landmarks (i.e., side branches) identified in the CTCA and NIRS-IVUS imaging data will be used to define segments of interest - i.e., segments that have been assessed by both imaging modalities and have a lumen diameter $\geq 2 \mathrm{~mm}$. CTCA analysis will be performed by an expert analysist using the QAngioCT 2.1 Research Edition software that enables automated extraction of the luminal centreline, semi-automated detection of the lumen and outer vessel wall borders and quantification of the plaque burden and composition. NIRS-IVUS analysis will be performed by an operator with expertise in intravascular image analysis blinded to the CTCA analysis using dedicated software (QIvus 3.1, Medis Medical Imaging Systems, Leiden, The Netherlands). The inter- and intra-observer reliability of the expert analyst who will perform the NIRS-IVUS and CTCA 
segmentation will be measured in 20 coronary arteries and reported. Lumen and outer vessel wall border detection will be performed in end-diastolic IVUS images while the presence of lipid component will be derived by the NIRS data that are displayed in a colour coded map called chemogram (yellow indicates increased probability and red low probability of lipid tissue). A metric of the lipid burden is the lipid core burden index (LCBI) which is computed as the fraction of the yellow pixels that correspond to lipid component divided by 1000 . In addition, for each $2 \mathrm{~mm}$ segments, the block chemogram is generated that provides a summary of the chemogram for this segment. The NIRS and IVUS imaging data will be used to characterise plaque composition and its phenotype.

\section{Vessel reconstruction, blood flow simulation and local haemodynamic forces assessment}

CTCA coronary artery segmentation will be performed using a module that automatically places the CTCA borders onto the luminal centreline and creates three dimensional (3D) surfaces of the lumen and outer vessel wall which can be processed with CFD techniques.(11) The segment of interest - i.e., the segment that has been assessed by both CTCA and NIRS-IVUS imaging - and its side branches will be reconstructed using this approach and blood flow simulation will be performed. An established and well validated methodology that relies on the fusion of NIRS-IVUS and the angiographic data will be used to reconstruct the segment of interest and its side branches (diameter $>1 \mathrm{~mm}$ ).(13) Blood flow will be estimated by measuring the number of the angiographic frames required for the contrast dye to travel from the start to the end of the reconstructed model, the volume of the coronary model and cine frame rate.(14) In the obtained geometries, blood flow simulation will be performed using the same blood flow and inflow and outflow boundary conditions for the NIRS-IVUS and CTC-based reconstructions and the ESS distribution will be estimated (Figure 1).

\section{Data post-processing}

We anticipate NIRS-IVUS imaging to be performed on average in 2.5 vessels per patient; from these 40 will be randomly selected and used to train algorithms for CTCA segmentation and plaque characterisation (training dataset) while the remaining data will be used for validation purposes (validation dataset). In the training set, the segments of interest will be divided in $2 \mathrm{~mm}$ segments and 
corresponding $2 \mathrm{~mm}$ segments will be identified in the CTCA and NIRS-IVUS models. In each segment the following metrics will be estimated in NIRS-IVUS: mean lumen area, vessel wall area, plaque area and burden, mean calcific area, the LCBI and the predominant ESS. In addition, each segment will be classified as lipid-rich or non-lipid rich according to the block chemogram.(15) Similarly, in the CTCA models the mean lumen area, outer vessel wall area, plaque area, plaque burden, calcific area and the mean predominant ESS will be estimated for every $2 \mathrm{~mm}$ segment and compared with the estimations of NIRS-IVUS. Several approaches will be tested to optimise the segmentation of the lumen and vessel wall borders in CTCA including machine learning techniques and the best will be selected. The adaptive Hounsfield unit cut-offs that best identify lipid and calcific tissue will be defined and spread-out vessel plots portraying the distribution of the lipid tissue in the CTCA models will be created and compared with the output of NIRS. The block chemogram in NIRS-IVUS will be used to identify the $2 \mathrm{~mm}$ CTCAderived lipid cut-off that enables accurate classification of the $2 \mathrm{~mm}$ segments as lipid or non-lipid rich. The accuracy of these cut-offs will be tested in the validation dataset. In addition, in the validation dataset the NIRS-IVUS data will be used to identify coronary lesions - defined as segments with a plaque burden $>40 \%$ in 3 consecutive frames.(16) For each lesion, its remodelling index, calcific and lipid component and plaque burden will be used to characterise their phenotype and classify them as: pathological intimal thickening/fibrotic plaques, fibro-calcific plaques, fibroatheromas, and calcified fibroatheromas $(6,17,18)$. The NIRS-IVUS lesion classification will be used as reference standard in order to assess the accuracy of CTCA in characterising lesion phenotype.

\section{Power calculation and statistical analyses}

The primary endpoint of the study is the ability of CTCA in detecting fibroatheromas. In the study of Garcia-Garcia et al that included 129 patients who underwent single vessel IVUS imaging,(16) 1.7 lesions were identified per patient. In the study of Puri et al. $45 \%$ of the detected lesions were classified as fibroatheromas based on histology.(6) In this study, a maximum 4mm LCBI >178, a remodelling index $>1.1$ and a plaque burden $>67 \%$ enabled detection of fibroatheromas with an excellent accuracy (c-index: 0.80). We anticipate that we will be able to perform NIRS-IVUS imaging in 2.5 coronary arteries per patient(19) and that CTCA imaging quality will be optimal in $93 \%$ of the studied 
patients. $(20,21)$ Therefore, a sample size of 70 patients will allow us to study 162 vessels, of which 120 vessels will be used for validation (203 lesions, of which 92 are fibroatheromas) purposes. This dataset is anticipated to provide $80 \%$ power to demonstrate that CTCA has a similar sensitivity to NIRSIVUS to detect fibroatheromas using the 5\% significant level (AUC of CTCA 0.71-0.89 and assuming that the true sensitivity of NIRS-IVUS is 0.80 ).

Secondary endpoints of the study are the accuracy of CTCA to identify: a) lipid-rich segments (using the block chemogram of NIRS-IVUS as the gold standard), and b) segments exposed to low ESS ( $<1 \mathrm{~Pa}$, using the ESS estimated in the NIRS-IVUS based reconstructions as the reference standard).

Correlation and linear regression analysis will be used to investigate the associations between mean lumen, outer vessel wall, plaque area, mean plaque burden and calcific tissue area estimated in the $2 \mathrm{~mm}$ segments in the NIRS-IVUS and CTCA models and mixed models with random intercept and slope will be used to correct for patient effects.

\section{Discussion}

Cumulative evidence has demonstrated that plaque composition determines its vulnerability and enables more accurate risk stratification. Intravascular imaging studies have shown that even single vesselplaque assessment provides useful prognostic information and identification of patients at risk. $(2,4,22$, 23) Despite the increased cost and the risk of complications associated with intravascular imaging, this strategy may have a value in selected populations with a high cardiovascular risk such as the patients admitted with an acute coronary syndrome where the event rate at 1 year is as high as $20 \%$. $(24,25)$ Modern submillimetre high resolution CTCA imaging allows non-invasive, complete assessment of the coronary tree and atherosclerotic plaques and offers a unique alternative in the study of atherosclerosis. Cumulative evidence has highlighted its value in assessing atheroma characteristics and identifying patients at risk for future events amongst individuals with no previous cardiac history, however its role in secondary prevention is yet to be explored at scale. $(20,26)$ This at least partially is attributed to the limited efficacy of the first generation CTCA scanners to assess complex atherosclerotic plaques and especially, lesions with an increased calcific component as well as the increased time required to process the CTCA imaging data. $(8,27,28)$ 
The objective of this study is to take advantage of the advances in CTCA imaging and use hybrid intravascular imaging to overcome well-known limitations of CTCA by developing a novel platform that will incorporate efficient methodologies for automated and reliable processing of the CTCA imaging data, coronary reconstruction and blood flow simulation in less than $1 \frac{1 / 2}{2}$ hour. To our knowledge this is the first prospective appropriately powered clinical study with a strict CTCA and 3vessel intravascular imaging protocol that aims to examine the efficacy of a 3 rd generation CT in assessing plaque characteristics in patients with advanced coronary artery disease. Moreover, in contrast to other studies, we aim not only to validate but also train segmentation and plaque characterisation algorithms, recognise and minimise the effect of common artefacts (i.e., calcium blooming artefact) on CTCA analysis, and thus assess more accurately plaque characteristics in patients with extensive/complex coronary artery disease. These advances will allow us to conduct studies that will examine the value of CTCA imaging in stratifying cardiovascular risk in patients with established coronary artery disease. This objective is timely as several new therapies that target vulnerable patients have been recently introduced in the clinical arena (i.e., Evolocumab, Alirocumab, prolonged dual antiplatelet treatment with low dose Ticagrelor),(29-31) others have been tested in large clinical studies and are likely to have applications in due course (i.e., Canakinumab)(32) and others are currently undergoing clinical evaluation in large randomised control trials [i.e., Colchicine (NCT02551094), Incliseran (NCT03705234)]. The above medications, however have significant side effects or are associated with an increased cost and thus they should be used only in well-defined high-risk patients. Unlocking the full potential of CTCA imaging in assessing atheroma characteristics and estimating the local hemodynamic forces - which in intravascular imaging studies appear to enable more accurate detection of vulnerable plaques and high-risk patients $(3,33)$ - is essential for the broad use of this modality in the clinical arena. This will allow identification of patients that are likely to sustain a cardiovascular event and benefit from an aggressive treatment of atherosclerosis.(34)

In addition, the platform that we envision to develop is expected to enhance the research applications of CTCA and facilitate the conduction of large clinical studies that will focus on the evaluation of the effects of new treatments on plaque growth. Moreover, this platform will enable assessment, in large asymptomatic population, the effects of local hemodynamic forces on the formation and destabilization 
of vulnerable plaques. This would allow us to understand the interplay between plaque morphology and flow patterns and their long term effects on plaque evolution.

\section{Limitations}

One of the limitations of the present study is the inclusion of patients with stable angina. Therefore, in contrast to other reports it will not be able to evaluate the efficacy of CTCA in detecting culprit lesions that have caused an acute coronary event.(35,36) In addition, we have excluded patients with severe chronic kidney disease, extensive coronary artery disease requiring surgical revascularisation and those who had previous coronary artery bypass grafting. These patients have advanced coronary artery disease with increased calcific burden. We acknowledge that, in these settings CTCA may be less accurate due to the blooming artefacts in assessing the lumen and outer vessel wall dimension and as well as quantification of the plaque burden.

\section{Ethical considerations}

The study protocol has been approved by the local and independent Research Ethics Committee (REC reference: 17/SC/0566) and will be conducted in agreement with the Declaration of Helsinki. All the adverse events (AE) that are likely to occur during the study will be assessed for severity, causality, seriousness and expectedness as per the sponsor's protocol; AE and serious adverse events (SAE) will be reported to the sponsor within five working days. If there is an increased event rate or concerns about patient safety during the conduction of the study, study termination will be considered.

\section{Dissemination}

We aim to complete recruitment by late-2019 and publications will be prepared in the mid of 2020 . Dissemination of the results will focus on publications in peer-reviewed journals in cardiovascular medicine and selected engineering journals as well as presentations at national and international meetings. The study has been also registered in a public registry (http://clinicaltrials.gov NCT035566440). 


\section{Sponsor}

The sponsor of the study is University College London; the study is jointly funded by British Heart Foundation (PG/17/18/32883), University College London Biomedical Resource Centre (BRC492B) and Rosetrees Trust (A1773).

\section{Summary}

Despite the wealth of data supporting the use for CTCA for the assessment of coronary luminal stenosis, the application of this modality for plaque characterisation and risk stratification remains limited. In this study, we aim to use intravascular imaging and in particular, NIRS-IVUS to train and validate segmentation and plaque characterisation methodologies and incorporate reconstruction and blood flow simulation algorithms in a fast and user-friendly software. This will enable the broad use of CTCA in the clinical arena for more accurate detection of high-risk patients and for the evaluation of the effects of emerging therapies on plaque progression.

\section{Disclosure Statement}

The authors have no conflicts of interests to declare. 


\section{References}

1. Stone GW, Maehara A, Lansky AJ, de Bruyne B, Cristea E, Mintz GS, et al. A prospective natural-history study of coronary atherosclerosis. The New England journal of medicine. 2011;364(3):226-35.

2. Waksman R. Assessment of coronary near-infrared spectroscopy imaging to detect vulnerable plaques and vulnerable patients. The Lipid-Rich Plaque study. Transcatheter Cardiovascular Theurapeutics. 2018;21-25 September(San Diego, CA).

3. Stone PH, Saito S, Takahashi S, Makita Y, Nakamura S, Kawasaki T, et al. Prediction of progression of coronary artery disease and clinical outcomes using vascular profiling of endothelial shear stress and arterial plaque characteristics: the PREDICTION Study. Circulation. 2012;126(2):172-81.

4. Prati F. Relationship between coronary plaque morphology of the left anterior descending artery and long term clinical outcome: the CLIMA study. EuroPCR. 2018;22-25 May:(Paris, France).

5. Kang SJ, Mintz GS, Pu J, Sum ST, Madden SP, Burke AP, et al. Combined IVUS and NIRS detection of fibroatheromas: histopathological validation in human coronary arteries. JACC Cardiovascular imaging. 2015;8(2):184-94.

6. Puri R, Madder RD, Madden SP, Sum ST, Wolski K, Muller JE, et al. Near-Infrared Spectroscopy Enhances Intravascular Ultrasound Assessment of Vulnerable Coronary Plaque: A Combined Pathological and In Vivo Study. Arteriosclerosis, thrombosis, and vascular biology. 2015;35(11):2423-31.

7. de Graaf MA, Broersen A, Kitslaar PH, Roos CJ, Dijkstra J, Lelieveldt BP, et al. Automatic quantification and characterization of coronary atherosclerosis with computed tomography coronary angiography: cross-correlation with intravascular ultrasound virtual histology. The international journal of cardiovascular imaging. 2013;29(5):1177-90.

8. Voros S, Rinehart S, Qian Z, Vazquez G, Anderson H, Murrieta L, et al. Prospective validation of standardized, 3-dimensional, quantitative coronary computed tomographic plaque measurements using radiofrequency backscatter intravascular ultrasound as reference standard in intermediate coronary arterial lesions: results from the ATLANTA (assessment of tissue characteristics, lesion morphology, and hemodynamics by angiography with fractional flow reserve, intravascular ultrasound and virtual histology, and noninvasive computed tomography in atherosclerotic plaques) I study. JACC Cardiovascular interventions. 2011;4(2):198-208.

9. Boogers MJ, Broersen A, van Velzen JE, de Graaf FR, El-Naggar HM, Kitslaar PH, et al. Automated quantification of coronary plaque with computed tomography: comparison with intravascular ultrasound using a dedicated registration algorithm for fusion-based quantification. European heart journal. 2012;33(8):1007-16.

10. Bourantas CV, Papadopoulou SL, Serruys PW, Sakellarios A, Kitslaar PH, Bizopoulos P, et al. Noninvasive Prediction of Atherosclerotic Progression: The PROSPECT-MSCT Study. JACC Cardiovascular imaging. 2016;9(8):1009-11.

11. Sakellarios A, Bourantas CV, Papadopoulou SL, Kitslaar PH, Girasis C, Stone GW, et al. The effect of coronary bifurcation and haemodynamics in prediction of atherosclerotic plaque development: a serial computed tomographic coronary angiographic study. Eurolntervention : journal of EuroPCR in collaboration with the Working Group on Interventional Cardiology of the European Society of Cardiology. 2017;13(9):e1084-e91. 
12. Lee JM, Choi G, Koo BK, Hwang D, Park J, Zhang J, et al. Identification of High-Risk Plaques Destined to Cause Acute Coronary Syndrome Using Coronary Computed Tomographic Angiography and Computational Fluid Dynamics. JACC Cardiovascular imaging. 2018.

13. Bourantas CV, Papafaklis MI, Athanasiou L, Kalatzis FG, Naka KK, Siogkas PK, et al. A new methodology for accurate 3-dimensional coronary artery reconstruction using routine intravascular ultrasound and angiographic data: implications for widespread assessment of endothelial shear stress in humans. Eurolntervention : journal of EuroPCR in collaboration with the Working Group on Interventional Cardiology of the European Society of Cardiology. 2013;9(5):582-93.

14. Bourantas CV, Raber L, Sakellarios A, Ueki Y, Zanchin T, Koskinas KC, et al. Utility of Multimodality Intravascular Imaging and the Local Hemodynamic Forces to Predict Atherosclerotic Disease Progression. JACC Cardiovascular imaging. 2019.

15. Gardner CM, Tan H, Hull EL, Lisauskas JB, Sum ST, Meese TM, et al. Detection of lipid core coronary plaques in autopsy specimens with a novel catheter-based near-infrared spectroscopy system. JACC Cardiovascular imaging. 2008;1(5):638-48.

16. Garcia-Garcia HM, Gomez-Lara J, Gonzalo N, Garg S, Shin ES, Goedhart D, et al. A comparison of the distribution of necrotic core in bifurcation and non-bifurcation coronary lesions: an in vivo assessment using intravascular ultrasound radiofrequency data analysis. Eurolntervention : journal of EuroPCR in collaboration with the Working Group on Interventional Cardiology of the European Society of Cardiology. 2010;6(3):321-7.

17. Garcia-Garcia HM, Mintz GS, Lerman A, Vince DG, Margolis MP, van Es GA, et al. Tissue characterisation using intravascular radiofrequency data analysis: recommendations for acquisition, analysis, interpretation and reporting. Eurolntervention : journal of EuroPCR in collaboration with the Working Group on Interventional Cardiology of the European Society of Cardiology. 2009;5(2):177-89.

18. Virmani R, Kolodgie FD, Burke AP, Farb A, Schwartz SM. Lessons from sudden coronary death: a comprehensive morphological classification scheme for atherosclerotic lesions. Arteriosclerosis, thrombosis, and vascular biology. 2000;20(5):1262-75.

19. Taniwaki M, Radu MD, Garcia-Garcia HM, Heg D, Kelbaek H, Holmvang L, et al. Longterm safety and feasibility of three-vessel multimodality intravascular imaging in patients with ST-elevation myocardial infarction: the IBIS-4 (integrated biomarker and imaging study) substudy. The international journal of cardiovascular imaging. 2015;31(5):915-26.

20. Versteylen MO, Kietselaer BL, Dagnelie PC, Joosen IA, Dedic A, Raaijmakers RH, et al. Additive value of semiautomated quantification of coronary artery disease using cardiac computed tomographic angiography to predict future acute coronary syndrome. Journal of the American College of Cardiology. 2013;61(22):2296-305.

21. Heo R, Park HB, Lee BK, Shin S, Arsanjani R, Min JK, et al. Optimal boundary detection method and window settings for coronary atherosclerotic plaque volume analysis in coronary computed tomography angiography: comparison with intravascular ultrasound. European radiology. 2016;26(9):3190-8.

22. Bourantas CV, Garcia-Garcia HM, Farooq V, Maehara A, Xu K, Genereux P, et al. Clinical and angiographic characteristics of patients likely to have vulnerable plaques: analysis from the PROSPECT study. JACC Cardiovascular imaging. 2013;6(12):1263-72.

23. Schuurman AS, Vroegindewey $M$, Kardys I, Oemrawsingh RM, Cheng JM, de Boer $S$, et al. Near-infrared spectroscopy-derived lipid core burden index predicts adverse 
cardiovascular outcome in patients with coronary artery disease during long-term follow-up. Eur Heart J. 2018;39(4):295-302.

24. Wallentin L, Becker RC, Budaj A, Cannon CP, Emanuelsson H, Held C, et al. Ticagrelor versus clopidogrel in patients with acute coronary syndromes. The New England journal of medicine. 2009;361(11):1045-57.

25. White HD, Ohman EM, Lincoff AM, Bertrand ME, Colombo A, McLaurin BT, et al. Safety and efficacy of bivalirudin with and without glycoprotein Ilb/Illa inhibitors in patients with acute coronary syndromes undergoing percutaneous coronary intervention 1-year results from the ACUITY (Acute Catheterization and Urgent Intervention Triage strategY) trial. Journal of the American College of Cardiology. 2008;52(10):807-14.

26. Otsuka K, Fukuda S, Tanaka A, Nakanishi K, Taguchi H, Yoshikawa J, et al. Napkin-ring sign on coronary $\mathrm{CT}$ angiography for the prediction of acute coronary syndrome. JACC Cardiovascular imaging. 2013;6(4):448-57.

27. Obaid DR, Calvert PA, Gopalan D, Parker RA, Hoole SP, West NE, et al. Atherosclerotic plaque composition and classification identified by coronary computed tomography: assessment of computed tomography-generated plaque maps compared with virtual histology intravascular ultrasound and histology. Circ Cardiovasc Imaging. 2013;6(5):655-64.

28. Maurovich-Horvat P, Schlett CL, Alkadhi H, Nakano M, Stolzmann P, Vorpahl M, et al. Differentiation of early from advanced coronary atherosclerotic lesions: systematic comparison of $\mathrm{CT}$, intravascular US, and optical frequency domain imaging with histopathologic examination in ex vivo human hearts. Radiology. 2012;265(2):393-401.

29. Bonaca MP, Braunwald E, Sabatine MS. Long-Term Use of Ticagrelor in Patients with Prior Myocardial Infarction. The New England journal of medicine. 2015;373(13):1274-5.

30. Sabatine MS, Giugliano RP, Keech AC, Honarpour N, Wiviott SD, Murphy SA, et al. Evolocumab and Clinical Outcomes in Patients with Cardiovascular Disease. The New England journal of medicine. 2017;376(18):1713-22.

31. Schwartz GG, Steg PG, Szarek M, Bhatt DL, Bittner VA, Diaz R, et al. Alirocumab and Cardiovascular Outcomes after Acute Coronary Syndrome. The New England journal of medicine. 2018;379(22):2097-107.

32. Ridker PM, Everett BM, Thuren T, MacFadyen JG, Chang WH, Ballantyne C, et al. Antiinflammatory Therapy with Canakinumab for Atherosclerotic Disease. The New England journal of medicine. 2017;377(12):1119-31.

33. Stone PH, Maehara A, Coskun AU, Maynard CC, Zaromytidou M, Siasos G, et al. Role of Low Endothelial Shear Stress and Plaque Characteristics in the Prediction of Nonculprit Major Adverse Cardiac Events: The PROSPECT Study. JACC Cardiovascular imaging. 2018;11(3):462-71.

34. Bourantas CV, Garcia-Garcia HM, Torii R, Zhang YJ, Westwood M, Crake T, et al. Vulnerable plaque detection: an unrealistic quest or a feasible objective with a clinical value? Heart. 2016;102(8):581-9.

35. Reith S, Battermann S, Hoffmann R, Marx N, Burgmaier M. Optical coherence tomography derived differences of plaque characteristics in coronary culprit lesions between type 2 diabetic patients with and without acute coronary syndrome. Catheter Cardiovasc Interv. 2014;84(5):700-7.

36. Ozaki Y, Okumura M, Ismail TF, Motoyama S, Naruse H, Hattori K, et al. Coronary CT angiographic characteristics of culprit lesions in acute coronary syndromes not related to plaque rupture as defined by optical coherence tomography and angioscopy. European heart journal. 2011;32(22):2814-23. 
Table 1. Study inclusion and exclusion criteria

\section{Inclusion criteria}

1. Patients with stable angina that have at least one obstructive lesion on elective diagnostic coronary angiography that require further assessment with intravascular imaging, invasive functional assessment or percutaneous coronary intervention

2. Age $18-75$ years old

3. Patient is able and willing to provide their written consent

\section{Exclusion criteria}

1. History of previous coronary artery bypass graft surgery

2. History of heart failure New York Heart Association (NYHA) IV or severe left ventricular systolic dysfunction $<30 \%$ regardless of patient symptom status

3. Documented allergy or inability to receive treatment with aspirin, heparin or thienopyridines

4. Patients that require surgical revascularisation following diagnostic angiogram

5. Renal impairment - estimated glomerular filtration rate of $<60 \mathrm{~mL} / \mathrm{min}$ at screening.

6. History of acute coronary syndrome in the last 3 months

7. History of heart transplantation

8. Life expectancy of less than one year

9. Extensive coronary artery disease (i.e. multiple chronic total occlusions) or torturous coronary anatomy that does not allow assessment with NIRS-IVUS imaging 


\section{Figure legends}

Figure 1. Example of the vessel segmentation, reconstruction and blood flow simulation for local haemodynamic forces assessment. (A). Coronary angiography of a right coronary artery (RCA). The segment of interest is defined by the most proximal and distal side branches seen on the NIRS-IVUS and CTCA. (B'). NIRS-IVUS cross-section showing an attenuated calcific plaque and (B") the corresponding chemogram indicating the lipid-rich plaque. (C). Coronary tree model of the same patient reconstructed using CTCA where the RCA has been highlighted. (D') CTCA cross-section showing lumen (yellow) and vessel wall (orange) annotations and plaque characteristics as well as (D") the longitudinal view of the RCA segmentation. (E). 3D reconstruction model of the RCA derived from the CTCA analysis showing luminal (light blue), vessel (green) surface and overlying plaques. (F). The ESS is a colour-coded display of the 3D model (blue colour indicates low ESS and red as high ESS). 


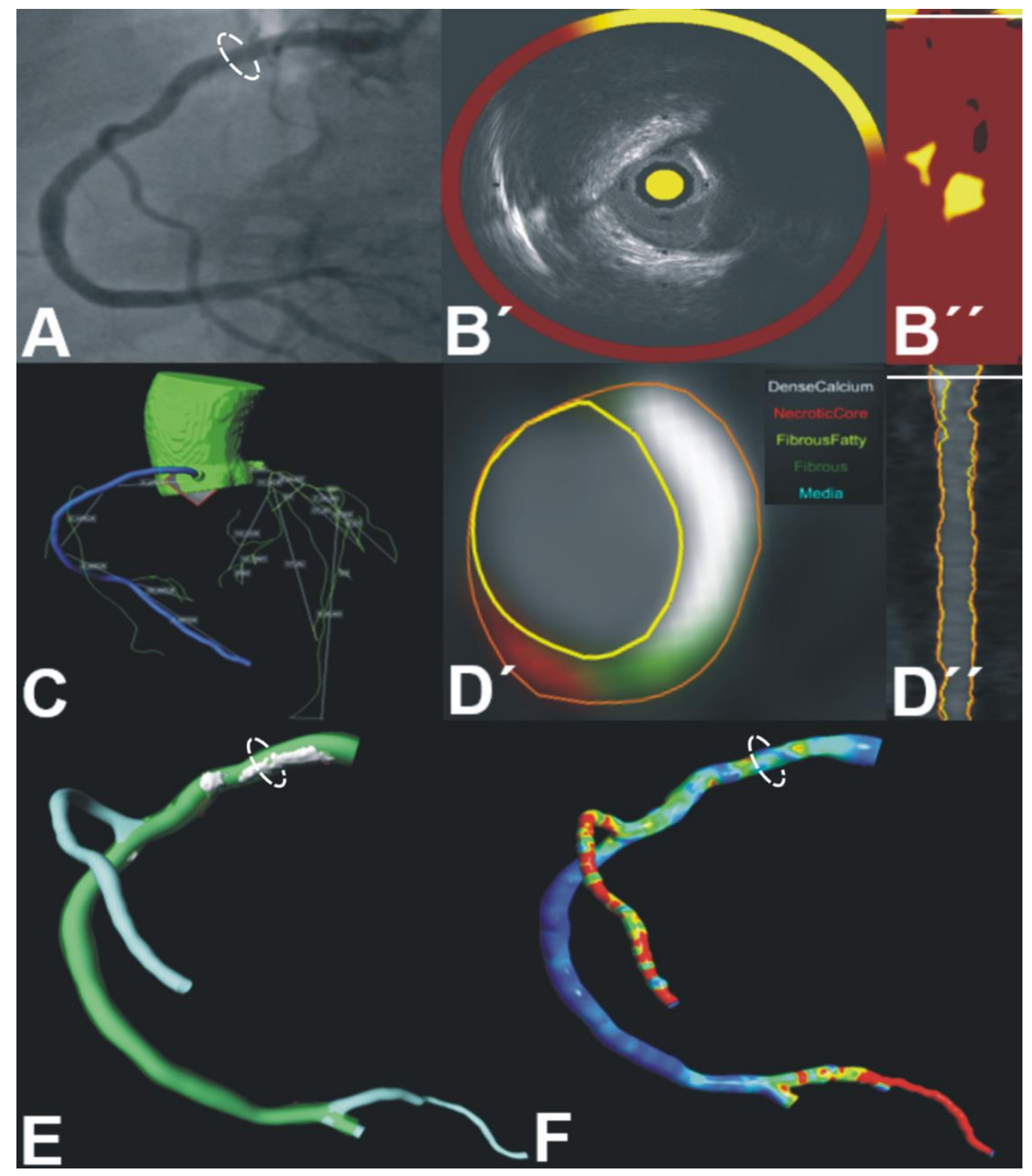

Figure 1 